\section{A Gynaecologist's Viewpoint}

\author{
By BEORN UYS, M.B., B.Ch., M.R.C.O.G.
}

The properly-trained patient will come into labour confident in her own ability to cope with the stringencies of this event, and emotional breakdown is very rare. As with any other student, she comes to look forward to her "final examination" as a time when she can prove herself, when she will see a successful culmination of her period of preparation and study; and the thought of failure does not seem to enter her head. True enough she wonders whether she will remember everything she has been taught at the critical moment, but she can be reassured that the vital elements of confidence and control will remain. Thus during her labour she will be largely self-sufficient and will place her confidence in her own abilities rather than leaning heavily on the medical practitioner or midwife attending her. The need for drugs is almost invariably less than in the untrained patient and dosages can be kept well within the safe limit. Her concentration in remembering what she is to do and in putting this into practice serves to keep her mind from wandering into dark alleys of apprehension. Not only are the techniques of relaxation and controlled breathing very effective in diminishing the amount of pain generated by uterine contraction and cervical dilatation but she is able to cope with the pain actually experienced with fortitude.

She has been told during antenatal instruction that during the earlier stages of labour sitting or walking is often preferable to lying down and the reasons for this have been explained. She has come to appreciate that labour is not just "something that happens to her" but an active process whose progress she can facilitate and she will voluntarily walk or sit up and take pride in her ability to do so. She will be interested in the progress of the labour and will want to know what stage she has reached. The absence of fear is remarkable; it is very true to say that an adult will seldom scream from pain but will do so from fear or panic. In properly trained patients one will perhaps encounter grunting and groaning but extremely seldom will hear screaming which occurs all too often in the untutored patient who is apt to lose control of herself when the going gets tough.

The doctor has the tremendous advantage with a trained patient of a labour which is usually shorter and smoother and of dealing with a rational patient who understands what is happening to her and can co-operate fully. Should he need to administer a drip, apply forceps or carry out any of the other common procedures he knows that this will not throw the patient into consternation because the reasons for these measures have been explained to her in the course of group lectures. To see a patient enjoying her labour and delivery is an experience which no doctor can readily forget.

\section{BACKACHE AND PELVIC PAIN}

Backache during and after pregnancy can arise from several different sources and causes but the commonest is undoubtedly the aching of lumbar and lower thoracic spinal muscles from incorrect posture and weakness of the rectus abdominis group. It must be carefully explained to the patient how drooping and poor tone of her abdominal muscles results in higher pelvic inclination and lumbar lordosis and in turn in a greater load being thrown on the abdominal muscles rather than on the pelvis. It is distressing to see how often a patient will enter her second pregnancy with rectus muscles still stretched and weak from her first pregnancy; she starts with a built-in disability and will often complain bitterly of bachache in the later months unless her problem can be corrected early on by means of vigourous exercises and postural instruction. Perhaps even worse is to see the appalling posture exhibited by many teenagers and to envisage the trouble they are going to encounter bearing even their first child. One is tempted to think that the early months of the first pregnancy is too late a time for instruction to begin and that prenatal classes should begin at school, but perhaps under a different name!

Low backache is a very common and distressing ailment in women in their late forties, and beyond, and is a source of despair to the family doctor and orthopaedic surgeon. This is all too often a late legacy of pregnancy due to correct posture not having been restored postnatally. It has perhaps not been sufficiently emphasised in the past that postnatal postural exercises do not apply only for a month or two after the baby is born but should continue for the rest of the life span. (Incidentally another common aftermath of inadequate postnatal exercise is prolapse of the uterus and vagina, manifesting itself usually after the menopause).

Pain will often arise in one or both sacroiliac joints and/or the symphysis pubis during pregnancy or soon after delivery. These joints are normally completely rigid and without movement in the non-pregnant state, but during pregnancy under the influence of hormones considerable slackening of the ligaments surrounding these joints takes place and consequently movement can occur and pain may result. The minor forms of sacroiliac strain are fortunately usually transient and are no contraindication to the continuation of prenatal exercises. Particular attention should be paid to the question of pelvic tilt as a near-vertical position of the pelvic brim will aggravate the load on the sacroiliac while a more horizontal inclination will relieve the strain.

When more than normal relaxation of the pelvic joints occurs there is excessive separation of the symphysis pubis and exaggerated movement at the sacroiliac joints. This condition has been termed pelvic arthropathy of pregnancy and can lead to severe pain. The pain may be of sudden onset and can be incapacitating, making even walking extremely difficult and painful. Occasionally severe pain makes its appearance only after labour. Patients suffering from this condition can obviously not continue with normal pre- and postnatal exercises. The recommended treatment is rest, mild muscle-toning exercise and support by a surgical belt, although the latter measure is difficult to apply and often unsatisfactory. At the present time no reason is evident for the excessive degree of relaxation of the pelvic joints exhibited by some patients and no specific treatment is known. Position during labour is important for them and excessive flexion and particularly the lithotomy position are to be avoided.

\section{CAESAREAN SECTION}

Over recent years there has been a change in approach to caesarean section. No longer is it an operation of last resort when vaginal delivery is impossible or excessively dangerous. More and more it is being realised that caesarean section is prefarable for both mother and baby to a difficult or prolonged labour. One has only to examine a patient after a difficult delivery and contrast the state of the pelvic organs with those of a woman who has had a caesarean section to appreciate how much less tissue trauma is involved in the latter procedure. A patient who has had a section will often have less pain and discomfort post-delivery than one who is left with a bruised pelvic floor and an extensive episiotomy. It is inevitable and right that caesarean section will be more and more freely employed in the future.

This matter affects fairly profoundly the antenate training of the patient. With intensive instruction and encouragement the patient comes to look forward to her labour and to "being present" at the delivery of her baby. It becomes in a sense a status symbol for her to do well in her labour and she can be intensely disappointed if she is deprived of this important event in her life. It is extremely difficult to know how to prepare the patient for the possibility that a caesarean section will be necessary.

In the patient with a borderline pelvis a trial labour is 
conducted, which means that labour is allowed to progress for some 8 to 12 hours before a decision is made as to doing a caesarean section or allowing labour to proceed and achieve vaginal delivery. If on the one hand the patient is told in the last few weeks of her pregnancy that she is to have a trial labour and that a section may be necessary she will be in some uncertainty and will not enter labour with full confidence that labour will progress in the normal way in which she has been instructed. On the other hand if she is not told of the possibility beforehand and is then told after some hours of labour that she now requires a section she is apt to imagine that some serious complication has suddenly arisen and however much she is reassured she will be apprehensive about the outcome for her baby. The emotional state of labour is not conducive to her being able at that stage to accept a rational explanation of what is happening.

A rewarding aspect of antenatal instruction is that the patient with a definitely contracted pelvis for whom vaginal delivery is not safe and who is told at 38 weeks that a caesarean section is definitely necessary will sometimes ask that she be allowed to have some hours of labour first. Through the medium of her instruction she has come to look forward to the experience of labour and the opportunity of proving herself and although she realises that section is inevitable does not want to be deprived completely of the labour.

In some centres in the United States and Canada there has in recent years been a strong swing towards natural childbirth, so much so that obstetricians have complained that where caesarean section becomes necessary they can have the utmost difficulty in persuading the patient to accept this.

The answer to the problem must lie in instructing patients at prenatal classes that caesarean section is not undertaken as a last desperate measure but rather as a means of forestalling complications and avoiding trauma to mother and baby. They should also be made aware that their prenatal training was not wasted should they not be able to deliver vaginally but that labour and delivery form but one part of the prenatal course and they will still have the advantages of posture, properly toned up muscles, correct breathing in the later months of pregnancy and an understanding of many aspects of childbearing. They should also not lose sight of the fact that postnatal exercises are as important after section as after vaginal delivery.

If the patient has this knowledge and has confidence that her doctor will choose the right course for her, little difficulty should be encountered in persuading her that caesarean section is the right course for her.

\section{THE SEXUAL ASPECT}

There are many aspects of pre- and postnatal instruction which are better imparted to a group than to an individual as the patient will then feel that the remarks are not being addressed to her personally and so she is able to assimilate the knowlegde without embarassment. This is particularly true of matters relating to sexual adjustment.

The delivery of a head of some 4 inches diameter through the urogenital hiatus which normally is of $1 \frac{1}{2}$ inch diameter will inevitably result in stretching of the vagina and of the muscles and ligaments surrounding it. The levator muscle will not spontaneously return to its former state but provided that it has not been overstretched or torn it can be restored to normal by means of assiduously-practised postnatal exercises. During delivery of the first infant episiotomy is frequently carried out as a means of avoiding overstretching of the muscle, but this leaves a scar which displays residual tenderness for some weeks or even months after delivery.

As sexual satisfaction and orgasm in the female are largely dependent on the integrity of the levator muscle it is hardly surprising that the patient with a stretched and relatively atonic levator and a tender scar to boot will not find much joy from the resumption of sexual relations when her baby is a month or 6 weeks old. Added to these difficulties are the fact that her interest has become very largely centred on the baby and that she is somewhat exhausted by the demands he makes on her.

It is therefore not very surprising that even the patient who has enjoyed good sexual responsiveness before her delivery will often complain of loss of interest, pain and absence of orgasm afterwards. Frequently she will be shy even to mention these difficulties to her doctor and may then never recover the normality she knew before in this regard. For the patient who was having difficulties before her pregnancy the problem is apt to be magnified tenfold.

There are also subtle hormonal changes that take place during pregnancy which may result in loss of sexual responsiveness at that time and there is frequently fear in the untutored that intercourse may be harmful to the baby. The enforced abstinence during the last month of pregnancy and for the first month after delivery can also cause a disruption in marital relations which is difficult to overcome. Added to these factors there may be the fear that another pregnancy may ensue sooner than is desired. For these various reasons loss of libido (normal sexual desire) is one of the commonest and most trying of postnatal complications.

Part of the answer lies in proper prenatal instruction as to the harmlessness of intercourse during pregnancy, emphasis on pelvic floor exercises pre- and postnatally, a healthy division of interest between husband and baby after confinement and proper contraceptive advice. The responsibility for these measures lies jointly with the doctor and the physiotherapist. These measures alone may be inadequate and then more detailed help must be given and perhaps the help of a psychologist sought.

\section{Psychological Principles Applied to Antenatal Training}

\author{
By alma e. HanNon, (M.A.) Psychology
}

Divergence of opinion exists amongst the proponents of antenatal training as to what constitutes a completely satisfactory programme for the preparation of childbirth. Successful performance during labour is considered the criterion for "satisfactoriness". Until a controlled investigation is undertaken where the results demonstrate a definitive relationship between method of training and performance during labour no claims for the superiority of one method over another can be made. Research in the efficacy of these different procedures would prove extremely laborious and almost since control of all the variables related to successful parturition would be difficult. How then can we assess the intrinsic value of any one of the training procedures? We may be prepared to assert that if it works, then it is successful. This is pure empiricism. Such an approach is permitted in a scientific discipline but it can prove sterile where the generation of new concepts, hypothesis or a theoretical model are concerned. The question should be "what makes it work?" This becomes a quest for the antecedents of the observed behaviour. "How does it work" on the other hand implies a quest for laws governing behaviour. If such an approach is adopted, we need explore no further, as the principles governing behaviour are already known to us. What we need to determine is how adequately they are applied to the area of antenatal care or training.

Antenatal training can be divided into three categories: Physical training through exercise and breathing; relaxation; and education in the physiology and mechanics of childbirth and labour. All three categories should be covered in the preparation for childbirth. The rationale for any antenatal procedure should be to equip the woman with responses 